\title{
So werden Leistungen bei ausländischen Patienten abgerechnet
}

- Für die ärztliche Behandlung von Patienten, die im Ausland krankenversichert sind und während ihres Aufenthalts in Deutschland erkranken, bestehen je nach Herkunftsland des jeweiligen Patienten unterschiedliche Abrechnungsmodalitäten. Patienten aus den Staaten des europäischen Wirtschaftsraums (EWR) und der Schweiz haben Anspruch auf alle Leistungen, die sich während ihres Aufenthalts in Deutschland als medizinisch notwendig erweisen. Um seinen Anspruch nachzuweisen, legt der Patient eine Europäische Krankenversicherungskarte oder eine Ersatzbescheinigung vor.

Bei allen anderen ausländischen Patienten, die z. B. auf Basis eines bilateralen Abkommens über soziale Sicherheit Anspruch auf Leistungen bei Krankheit und Mutterschaft haben, können ebenfalls bei einem Aufenthalt in Deutschland ärztliche Leistungen abgerechnet werden. Diese Patienten müssen sich aber mit einem An- spruchsnachweis ihrer heimischen Krankenkasse zunächst an eine von ihnen gewählte deutsche Krankenkasse wenden.

\section{MMW Kommentar}

Konkret bedeutet das, dass ein Patient aus einem EWR-Staat oder der Schweiz zur Behandlung eine gültige Europäische Krankenversicherungskarte (EHIC) oder eine Ersatzbescheinigung (PEB) sowie einen Identitätsnachweis (Reisepass oder Personalausweis) vorlegen muss. EHIC bzw. die PEB und der Identitätsnachweis sollten zweimal kopiert werden. Alternativ können die Daten auch in das Muster 80 (Dokumentation des Behandlungsanspruchs von im Ausland Versicherten) eingetragen, mit dem Arztstempel versehen und unterschrieben werden. Der Patient muss das Muster 81 (Erklärung des $\mathrm{Pa}$ tienten) ausfüllen und unterschreiben, wobei er die von ihm gewählte deutsche Krankenkasse angeben muss. Die Kopie der EHIC/PEB und die Kopie des Identitätsnachweises (al- ternativ Muster 80) sowie das Muster 81 müssen möglichst schnell an die vom Patienten gewählte Krankenkasse geschickt werden. Die Zweitkopie verbleibt in der Praxis und muss zwei Jahre aufbewahrt werden. Die Kosten für die Behandlung werden über die zuständige KV nach den Regelungen des Ersatzverfahrens (nach EBM) abgerechnet. Dazu muss ein Abrechnungsschein (Muster 5) ausgefüllt werden. Im Adressfeld erscheinen Name, Vorname und Geburtsdatum sowie die gewählte Krankenkasse, im Feld "Status" die Ziffer "10007". Die Fotokopien können nach Nr. 40144 EBM, die Portokosten nach Nr. 40120 EBM berechnet werden. Die Verordnung von Arznei-, Heil- und Hilfsmitteln kann auf den üblichen Formularen erfolgen. Ebenso eine Verordnung von Krankenhausbehandlung oder Überweisungen sowie die Bescheinigung der Arbeitsunfähigkeit. Der Patient erhält hier das Original sowie die Durchschrift zur Vorlage beim Arbeitgeber und der Krankenkasse.

\section{ZI-Report: vertragsärztliche Leistungen deutlich unterfinanziert}

\footnotetext{
- Das Zentralinstitut für die kassenärztliche Versorgung in Deutschland (ZI) erhebt regelmäßig betriebswirtschaftliche Daten in den Praxen und wertet sie aus. Danach sind die Umsätze der Praxisinhaber zwischen 2007 und 2009 im Schnitt pro Jahr um 4,5\% gestiegen. Der Verdienst aus rein vertragsärztlicher Tätigkeit lag mit durchschnittlich 98300 Euro im Jahr 2009 aber weiterhin deutlich unter dem Oberarztgehalt im Krankenhaus. Ein angestellter Oberarzt konnte im Jahr 2007 mit einem Jahresgehalt von 105600 Euro rechnen, im Jahr 2009 mit 114500 Euro.
}

\section{MMW Kommentar}

Ein Grund für das magere Ergebnis sind die Betriebskosten der Praxen, die zwischen 2007 und 2009 durchschnittlich um 2,8\% gestiegen sind. Auffällig ist dabei, dass der Kostenanstieg in Gemeinschaftspraxen (3\%) deutlich über dem der Einzelpraxen (1,8\%) lag. Der Unterschied resultiert im Wesentlichen daraus, dass Gemeinschaftspraxen mehr investieren als Einzelpraxen. Niedergelassene Vertragsärzte und -psychotherapeuten arbeiteten nach eigenen Angaben im Jahr 2009 durchschnittlich
50 Stunden pro Woche. Davon entfielen 36 Stunden (72\%) auf den direkten Patientenkontakt (Beratung, Untersuchung und Behandlung), 8 Stunden (16\%) auf ärztliche Tätigkeit ohne Anwesenheit von Patienten (Dokumentation, Gutachtenerstellung, Fallkonferenzen, Fachlektüre) und 2 Stunden auf Bereitschaftsdienste (4\%). Für Praxismanagement (Führungsaufgaben und Praxisorganisation) fielen rund 4 Stunden (8\%) an. 